\title{
Evaluación de la fuerza de anclajes de andamios de fachada
}

\section{Evaluation of the strenght of anchorages of facade scaffoldings}

\author{
S. González-Rodrigo ${ }^{(*)}$, F. González-Yunta ${ }^{(*)}$, M.N. González-García ${ }^{(*)}, \underline{\text { A. Cobo }}^{(*)}$
}

\section{RESUMEN}

La resistencia de los anclajes a fachada de las protecciones colectivas (PC) o de los medios auxiliares de edificación (MAE) constituye un punto esencial de la estabilidad de esos elementos, que en muchos casos se desconoce. Se han realizado numerosos estudios acerca de la resistencia y del comportamiento de los anclajes metálicos a estructuras de hormigón. Sin embargo apenas existen trabajos que analicen la resistencia de anclajes a fábricas de albañilería y mucho menos a fábricas de albañilería con decenios de antigüedad en situaciones reales: edificios en los que hay que intervenir y es preciso anclar PC y MAE a sus fábricas.

En este trabajo se ha evaluado la resistencia a arrancamiento de cáncamos insertados en tacos de plástico alojados en fábricas de albañilería de edificación ya construidas. Los resultados obtenidos se han comparado con los de otros investigadores y han permitido valorar las prescripciones de la norma UNE-EN 12810-1 relativas a la disposición y separación de anclajes de los andamios a fachada.

Palabras clave: Anclajes; andamios; albañilería; seguridad; construcción.

\section{SUMMARY}

The resistance of the anchors to the facade of collective protection (CP) or building aids (MAE) is an essential point of the stability of these elements, which in many cases is unknown. There have been numerous studies on resistance and behavior of the braces to concrete structures. But hardly any studies analyzing the strength of masonry anchors, much less masonry with decades of service in real situations: buildings that have to intervene and must anchor CP and MAE to its factories.

In this study we have evaluated the pullout resistance of cancer plastic plugs inserted into staying in masonry building already built. The results were compared with those of other investigators and allowed to assess the requirements of the standard UNE-EN 1281O-1 regarding the disposition and removal of scaffolding anchors to the facade.

Keywords: Anchors; scaffolding; masonry; safety; construction.

(*) Universidad Politécnica de Madrid, (España).

Persona de contacto/Corresponding author: alfonso.cobo@upm.es (A. Cobo)

Cómo citar este artículo/Citation: González-Rodrigo, S., González-Yunta, F., González-García, M.N., Cobo, A. (2014). Evaluación de la fuerza de anclajes de andamios de fachada. Informes de la Construcción, 66(533): e013, doi: http://dx.doi.org/10.3989/ic.11.141.

Licencia/License: Salvo indicación contraria, todos los contenidos de la edición electrónica de Informes de la Construcción se distribuyen bajo una licencia de uso y distribución Creative Commons Reconocimiento no Comercial 3.o. España (cc-by-nc). 


\section{INTRODUCCIÓN}

Uno de los puntos esenciales del proceso de verificación o dimensionamiento de un andamio lo constituye la resistencia al arrancamiento de los anclajes del mismo a la fachada del edificio sobre la que se trabaja.

El método de cálculo del anclaje se establece en base al cumplimiento de la siguiente ecuación [1]:

$$
S_{d} \leq R_{d}
$$

Donde $S_{d}$ es la solicitación sobre el anclaje como consecuencia de las cargas horizontales que actúan sobre el andamio y $R_{d}$ representa la resistencia del anclaje al arrancamiento o al esfuerzo cortante en función de si las fuerzas exteriores son perpendiculares o paralelas a fachada.

La solicitación sobre el anclaje se puede conocer con precisión utilizando las normas europeas que tratan el cálculo de andamios.

Lamentablemente, la resistencia del anclaje se desconoce en la mayoría de las ocasiones. Existen recomendaciones de tipo general que ofrecen valores límite para la resistencia al arrancamiento de anclajes. El Sindicato de Encofradores de Francia indica que los anclajes realizados utilizando tacos de plástico no deberían estar dimensionados para cargas superiores a $0,30 \mathrm{kN}$ (1). En otros casos se ofrecen recomendaciones de tipo genérico acerca del número de anclajes a realizar en función de la superficie del andamio, pero sin precisar valores de carga de arrancamiento (2) o del tipo de anclaje a utilizar en función del tipo de fachada (3). Se conocen trabajos bien documentados de anclajes realizados con elementos metálicos (4) (5), pero prácticamente no existen trabajos con anclajes realizados con tacos de plástico (6).

\section{OBJETIVOS}

El objetivo de este trabajo es valorar las disposiciones usuales de anclajes de andamios de fachada a fábricas de albañilería. Para ello se ha obtenido la resistencia de arrancamiento de anclajes realizados con tacos de plástico a obras de albañilería a partir de los datos experimentales existentes en la bibliografía y de los valores obtenidos por los autores de este trabajo sobre fábricas de ladrillo macizo efectuadas en Madrid en la década de 1930. Los valores obtenidos por ambos procedimientos se han comparado con la solicitación de un andamio tipo revestido con lona y calculado conforme a las normas UNE-EN.

\section{COMPORTAMIENTO DE ANCLAJES EN OBRAS DE ALBAÑILERÍA}

Del mismo modo a como ocurre en el hormigón, la capacidad de carga de un anclaje de plástico en obras de albañilería, depende de la fuerza de expansión que se puede producir. El nivel de la fuerza de expansión depende fundamentalmente de la resistencia a compresión del material de la fábrica, a menor resistencia, la fuerza de expansión es también menor. En ladrillos y bloques perforados, la fuerza de expansión se ejerce sobre la parte maciza de las piezas, lo que supone la necesidad de la suficiente superficie de contacto en la zona taladrada para poder transferir la fuerza del anclaje. Tanto en los anclajes sobre piezas macizas como en los efectuados sobre piezas aligeradas, en la mayoría de las ocasiones la forma de fallo es por arrancamiento (7).

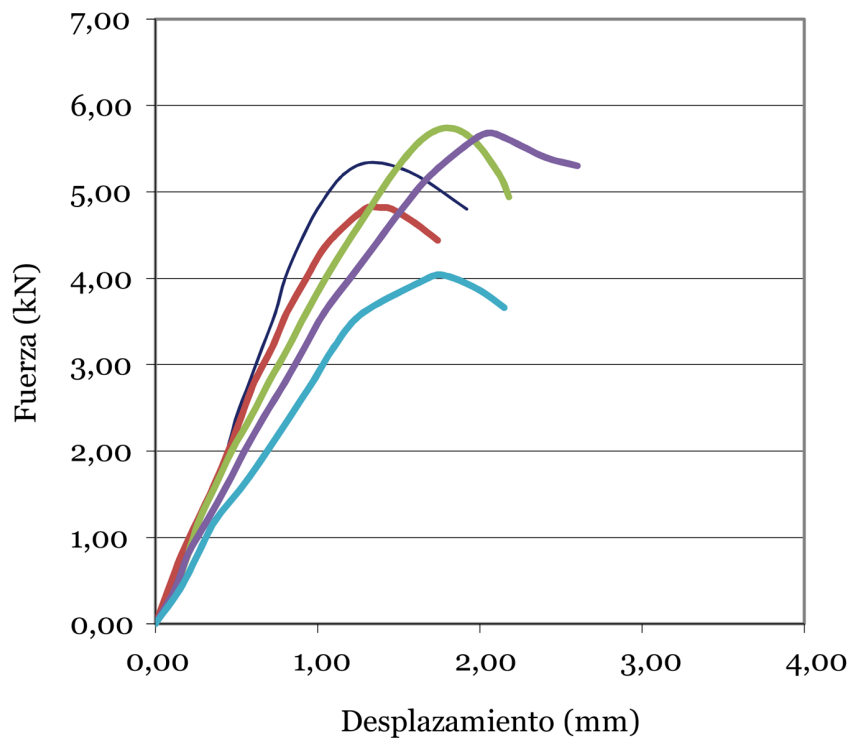

Figura 1. Comportamiento carga desplazamiento de anclajes en fábricas de ladrillo macizo (19MPa).

El comportamiento frente a arrancamiento de anclajes de plástico en obras de albañilería fabricadas con ladrillo macizo es muy similar al del hormigón. En la Figura 1 se muestran los ensayos realizados por Fischerwerke (8) en los que se puede apreciar un comportamiento lineal prácticamente hasta carga máxima, una vez alcanzada ésta, el colapso se produce con desplazamientos muy pequeños. El modo de fallo se produce por arrancamiento. El diámetro del anclaje utilizado en los ensayos fue de $10 \mathrm{~mm}$, su longitud $50 \mathrm{~mm}$ y la resistencia a compresión del ladrillo 19 MPa. La carga de arrancamiento se sitúa entre 4,o kN y $5,7 \mathrm{kN}$.

Los anclajes realizados sobre fábricas con piezas perforadas muestran resultados con mayor dispersión que los realizados sobre elementos macizos (Figura 2). Además la carga a arrancamiento disminuye de manera muy importante y los desplazamientos últimos son superiores, respecto de los resultados sobre anclajes en fábricas de ladrillo macizo (9).

La resistencia a compresión de la fábrica influye notablemente en la carga de fallo. En la Figura 3 se muestra el diagrama

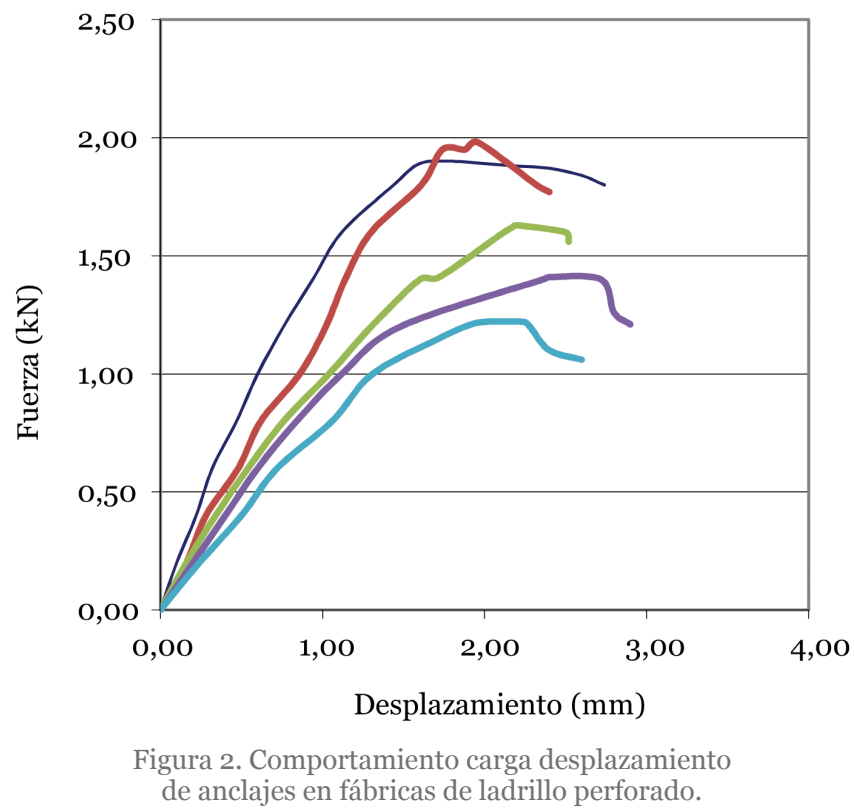


carga-desplazamiento de anclajes de $14 \mathrm{~mm}$ de diámetro y 70 $\mathrm{mm}$ de profundidad realizados sobre fábrica de ladrillo macizo de 5,4 MPa de resistencia a compresión (10).

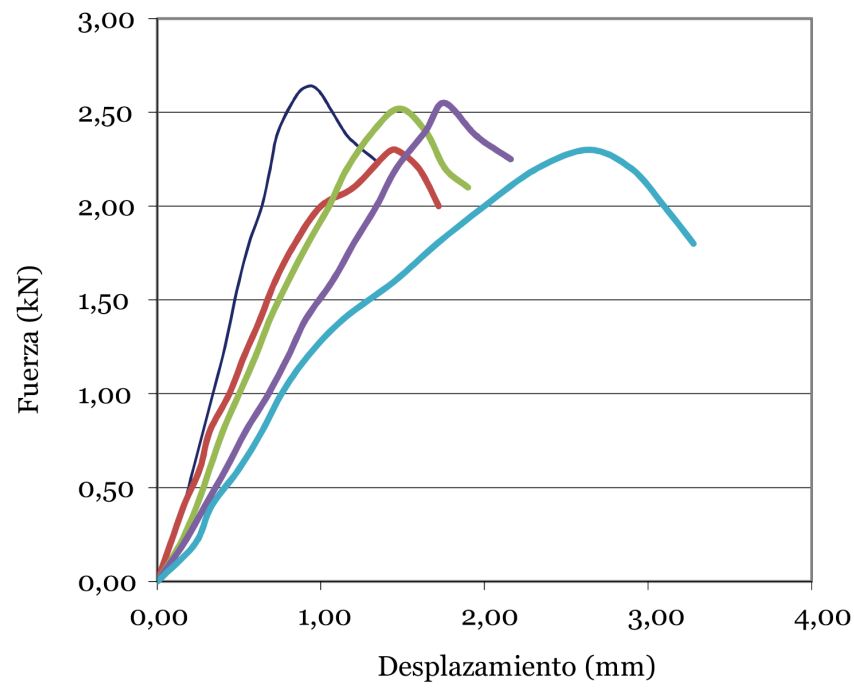

Figura 3. Comportamiento carga desplazamiento de anclajes en fábricas de ladrillo macizo $(5,4 \mathrm{MPa})$.

La comparación de estos valores con los de la Figura 1 muestra como a pesar del mayor diámetro y longitud de este anclaje, la fuerza de arrancamiento es mucho menor. Debido a su comportamiento menos rígido, los desplazamientos antes del fallo son iguales a los de la Figura 1 o incluso superiores.

En fábricas de ladrillo perforado, la resistencia del anclaje es difícilmente predecible, dependiendo su valor de la proporción de superficie maciza ocupada por el anclaje. En la Figura 4 se muestran los valores experimentales obtenidos de fuerza de anclaje en función de la superficie maciza ocupada por éste (11). Los valores numéricos indican la carga de arrancamiento en $\mathrm{kN}$.

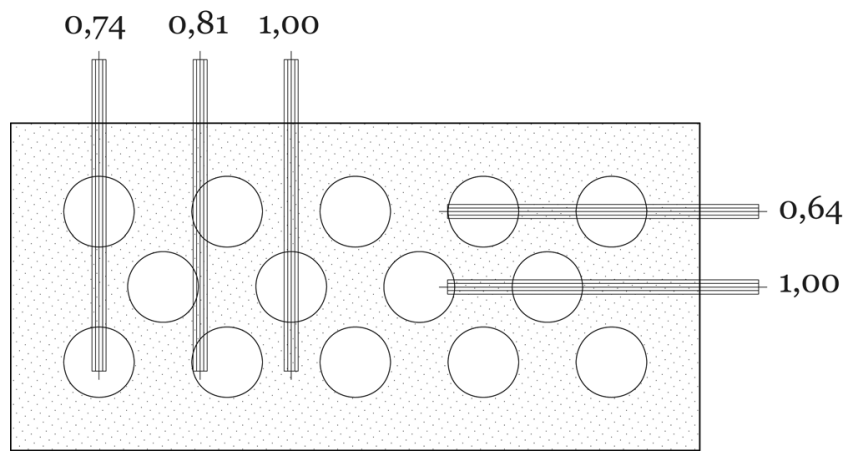

Figura 4. Resistencia relativa de anclajes en fábricas de ladrillo perforado.

Los autores de este trabajo han desarrollado una campaña experimental para obtener la fuerza de arrancamiento de anclajes de cáncamos de acero alojados en tacos de plástico sobre fábricas de ladrillo macizo construidas en Madrid en la década de 1930. Se han empleado cáncamos de acero de $120 \mathrm{~mm}$ de longitud y $10 \mathrm{~mm}$ de diámetro alojados en tacos de plástico de $70 \mathrm{~mm}$ de longitud, solución habitualmente empleada en España como amarre de andamios a fachada. Se han realizado doce ensayos de arrancamiento alojando el taco en el centro del ladrillo. El valor medio de arrancamiento obtenido ha sido de $6,2 \mathrm{kN}$, los valores máximo y mínimo han sido de $8,5 \mathrm{kN}$ y $5,3 \mathrm{kN}$.

\section{NORMAS UNE-EN RELATIVAS AL CÁLCULO DE ANDAMIOS}

A diferencia de lo que ocurre con otros países de la Unión Europea como el Reino Unido (British Standard), Francia (AFNOR) o Alemania (DIN), en España no existe normativa específica de obligado cumplimiento acerca del cálculo de andamios.

Como cuerpo normativo de referencia en el cálculo de las estructuras de andamio se cuenta con un conjunto de normas UNE, los Eurocódigos Estructurales y el Código Técnico de la Edificación. El artículo 194 del Convenio colectivo de la Construcción (12), de acuerdo con lo dispuesto en el RD 1801/2003, establece que cuando no exista disposición normativa de obligado cumplimiento aplicable, o ésta no cubra todos los riesgos o categorías de riesgos de este tipo de andamio, para garantizar el nivel de seguridad se tengan en cuenta la normas UNE existentes.

Las normas UNE-EN 12810 partes 1 y 2 (13) (14) y las normas UNE-EN 12811 Partes 1, 2 y 3 (15) (16) (17) forman un grupo de normas relativas al cálculo de andamios. El conjunto de estas cinco normas viene a sustituir al Documento de Armonización HD 1000 (18).

En las normas UNE-EN 12810-1, UNE-EN 12810-2 y UNEEN 12811-1 se indican los requisitos generales de diseño. La información se ofrece repartida en las tres normas y a menudo aparece muy desordenada.

La norma UNE-EN 12811-2 proporciona una guía sobre los materiales utilizados habitualmente en la fabricación de andamios. En concreto se ofrecen características acerca de los siguientes materiales: acero, aleaciones de aluminio, hierro fundido, madera y materiales basados en la madera. También se dan requisitos para la soldadura y límites a la corrosión y otros deterioros.

La norma UNE-EN 12811-3 especifica las reglas que se han de aplicar en los ensayos de carga sobre los materiales, configuraciones y componentes. En la norma se indica la documentación necesaria y la evaluación de los resultados de los ensayos.

La norma EN 12811-1 indica las acciones que se deben tomar en consideración para el cálculo de la estructura del andamio:

- Carga permanente. Incluye el peso propio de la estructura del andamio y de todos sus componentes.

- Carga variable. Incluye la carga en el área de trabajo, la carga sobre la protección lateral, la carga de viento y, si procede, la carga de nieve.

- Carga accidental. Es la correspondiente a la protección lateral (carga descendente).

La carga originada por el viento también viene definida en EN 12811-1. Su valor se obtiene como el producto de cuatro factores:

- Presión del viento.

- Superficie expuesta. 
- Coeficiente de sitio.

- Coeficiente de fuerza aerodinámica.

El valor de la superficie expuesta, el coeficiente de sitio y el coeficiente de fuerza aerodinámica depende de si el andamio está revestido o no. El cálculo de la estructura del andamio debe realizarse para dos direcciones de viento:

- Viento paralelo a la fachada sobre la que se asienta el andamio.

- Viento perpendicular a la fachada sobre la que se asienta el andamio.

Asimismo, la norma distingue dos situaciones de viento:

- Máxima carga de viento.

- Carga de viento de servicio.

\subsection{Andamio de trabajo sin revestir}

La fuerza resultante del viento en $\mathrm{kN}$, se obtiene a partir de la ecuación [2]:

$$
F=c_{s} \sum_{i}\left(c_{f, i} \cdot A_{i} \cdot q_{i}\right)
$$

Siendo:

$F$ : fuerza resultante del viento.

$c_{f, i}:$ Coeficiente de fuerza aerodinámica para el componente del andamio $i$.

$A_{i}$ : Área de referencia del componente del andamio i.

$q_{i}$ : Presión dinámica actuando sobre el componente del andamio $i$.

$c_{s}$ : Coeficiente del sitio.

A continuación se distinguen dos situaciones, en función de si el andamio está revestido o no.

\subsubsection{Coeficiente de fuerza aerodinámica $c_{f, i}$}

El valor del coeficiente de fuerza aerodinámica $c_{f, i}$ debe tomarse igual a 1,3 para todas las áreas proyectadas, incluyendo plataformas, rodapiés y el área nominal que se define a continuación. Con objeto de dar un margen a los equipos o materiales que están en el área de trabajo, debe asumirse un área de referencia nominal a su nivel en toda su longitud. Éste área debe tener $200 \mathrm{~mm}$ de altura medida desde el nivel del área de trabajo e incluye la altura del rodapié, para la condición de máxima carga de viento. Para la carga de viento en servicio, la altura del área será de $400 \mathrm{~mm}$. Se debe asumir que las cargas resultantes de la presión del viento en éste área actúan en el nivel del área de trabajo.

\subsubsection{Coeficiente del sitio $c_{s}$}

Para fuerzas de viento perpendiculares a la fachada, el coeficiente del sitio $\mathbf{c}_{s}$ se puede obtener de la Figura 5, tomada de EN 12811-1.

$\varphi_{B}$ es la relación de solidez y viene dada por la siguiente ecuación [3]:

$$
\varphi_{B}=\frac{A_{B, n}}{A_{B, g}}
$$

Siendo:

$A_{B, n}$ : Área neta de la fachada (deduciendo las aberturas).

$\mathrm{A}_{B, g}$ : Área total de la fachada.

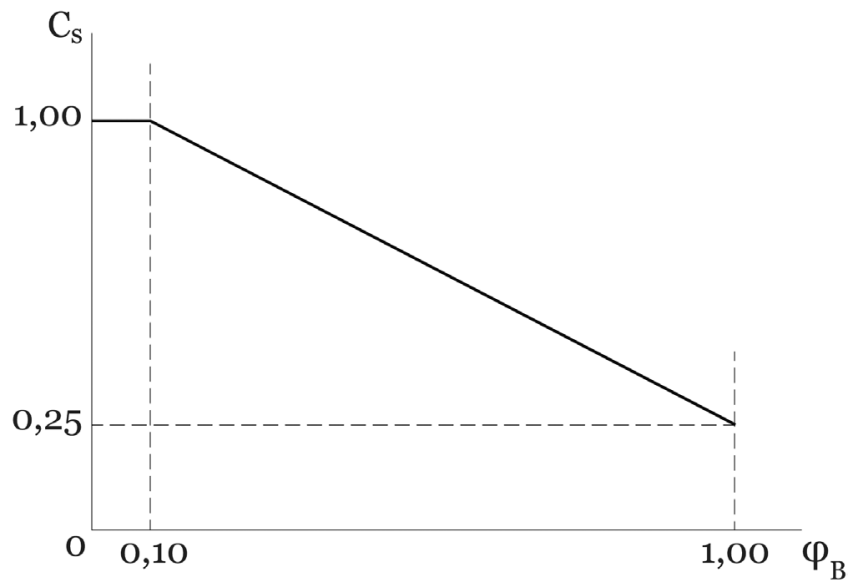

Figura 5. Coeficiente de sitio para andamios delante de fachada y con fuerzas de viento perpendiculares a ella.

Para fuerzas de viento paralelas a la fachada, el coeficiente del sitio $\mathbf{c}_{\boldsymbol{s}}$ se tomará igual a la unidad.

\subsubsection{Presión debida al viento}

En condiciones de viento máximo, se supone una presión debida al viento de $800 \mathrm{~N} / \mathrm{m}^{2}$ en la base del andamio que aumenta de forma lineal de modo que alcanza un valor de $1100 \mathrm{~N} / \mathrm{m}^{2}$ a la altura de 24,0 metros. En la Figura 6 (de EN 12810-1) se ofrece la ley hasta 25,5 metros. Esta presión actúa sobre la superficie proyectada del andamio.

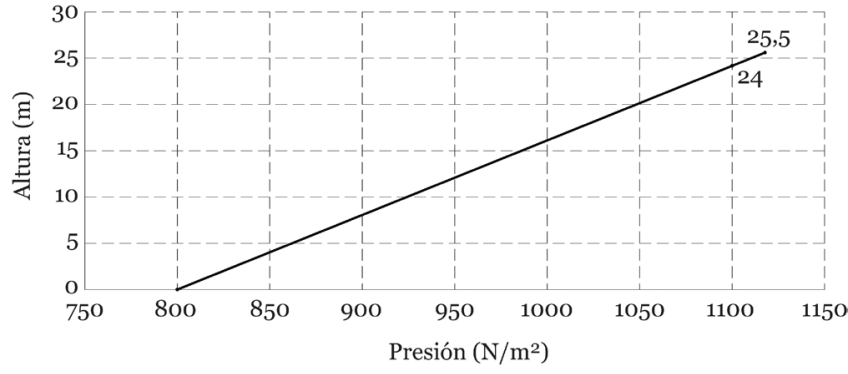

Figura 6. Presión de viento máximo.

En condiciones de viento en servicio, se supone una presión debida al viento de $200 \mathrm{~N} / \mathrm{m}^{2}$ uniformemente repartida en la superficie proyectada del andamio. Para este cálculo es necesario añadir una superficie nominal a la superficie $A_{\perp r}{ }^{1}$ y $A_{\mathrm{II} r}{ }^{2}$. Esta superficie tiene en cuenta la obstrucción al viento producida por pilas de materiales u otros elementos que descansan sobre las plataformas. Esta superficie nominal solamente debe suponerse sobre la superficie de la plataforma.

\footnotetext{
${ }^{1}$ Proyección de la superficie total de todos los elementos redondos y planos, sobre un plano perpendicular a la fachada (en $\mathrm{m}^{2}$ ).

2 Proyección de la superficie total de todos los elementos redondos y planos sobre un plano paralelo a la fachada (en $\mathrm{m}^{2}$ ).
} 


\subsection{Andamio de trabajo revestido}

La fuerza resultante del viento en $\mathrm{kN}$, se obtiene a partir de la ecuación [4]:

$$
F=c_{s} \sum_{i}\left(c_{f, i} \cdot A_{i} \cdot q_{i}\right)
$$

Siendo:

$F$ : fuerza resultante del viento.

$c_{f i}$ : Coeficiente de fuerza aerodinámica para el recubrimiento. $A_{i}$ : Área de referencia del recubrimiento.

$q_{i}$ : Presión dinámica actuando sobre la sección del recubrimiento $i$.

$c_{s}$ : Coeficiente del sitio.

\subsubsection{Coeficiente de fuerza aerodinámica $\mathrm{c}_{f}$}

Se obtiene de la Tabla 1 en función del tipo de recubrimiento (red o lona) y para viento normal $(\perp)$ y paralelo (II) al recubrimiento.

Tabla 1.

Coeficientes de fuerza aerodinámica para andamios revestidos.

\begin{tabular}{|l|c|c|}
\hline & $\mathbf{c}_{f \perp}$ & $\mathbf{c}_{\boldsymbol{f I I}}$ \\
\hline Red & 1,3 & 0,3 \\
\hline Lona & 1,3 & 0,1 \\
\hline
\end{tabular}

4.2.2. Área de referencia, $A$

Para las acciones de viento normales y paralelas al plano del recubrimiento, el área total del recubrimiento constituye el área de referencia, $A$.

Puede no considerarse el viento que actúa de forma normal al plano de las áreas de recubrimiento de los componentes del andamio u objetos detrás del recubrimiento (lona o red).

\subsubsection{Coeficiente de sitio, $c_{s}$}

Depende de la relación de solidez $\varphi_{B}$ que viene dada por la siguiente ecuación [5]:

$$
\varphi_{B}=\frac{A_{B, n}}{A_{B, g}}
$$

Siendo:

$A_{B, n}$ : Área neta de la fachada (deduciendo las aberturas). $A_{B, g}$ : Área total de la fachada.

El valor de $c_{s}$ se obtiene de la Figura 7, con las siguientes consideraciones:

- Para las redes se utiliza la curva 1.

- Para las redes en las que $c_{f \perp}$ es mayor que 0,8 , dicha red debe considerarse como lona para calcular el coeficiente de sitio.

- Para lonas se utiliza la curva $2\left(c_{s}=1,0\right)$.

- El coeficiente $\mathbf{c}_{\boldsymbol{s}}$ para el cálculo de la tensión de las fuerzas de anclaje de las uniones del andamio en el lado de sotavento, puede tomarse de la curva 3.

- Para el cálculo de las cargas de viento en el área del extremo de un tramo de un andamio de trabajo, $\boldsymbol{c}_{\boldsymbol{s}}$ debe ser igual a 1,0.

4.2.4. Cálculo de la fuerza paralela a la fachada. [6]

$$
F_{\mathrm{II}}=W \cdot c_{\mathrm{II}} \cdot\left[A_{\perp r}+\sum_{1}^{n} \frac{A_{\perp s} c_{f}}{1,2}\right]
$$

$W$ : Presión que ejerce el viento $\left(\mathrm{N} / \mathrm{m}^{2}\right)$.

$c_{\mathrm{II}}$ : Coeficiente de presión global para las fuerzas paralelas al andamio (de valor 1,1).

$A_{\perp r}$ : Proyección de la superficie total de todos los elementos redondos y planos, sobre un plano perpendicular a la fachada $\left(\right.$ en $\left.\mathrm{m}^{2}\right)$.

$A_{\perp s}$ : Proyección de la superficie total de las secciones transversales particulares (las no incluidas en $\mathrm{A}_{\downarrow r}$ ) sobre un plano perpendicular a la fachada $\left(\mathrm{en}^{2}\right)$.

$c_{f}$ : Coeficiente de presión para las secciones transversales.

4.2.5. Cálculo de la fuerza perpendicular a la fachada. [7]

$$
F=W \cdot c_{\perp} \cdot\left[A_{\mathrm{II} r}+\sum_{1}^{n} \frac{A_{\mathrm{IIs}} \cdot c_{f}}{1,2}\right]
$$

$W$ : Presión que ejerce el viento $\left(\mathrm{N} / \mathrm{m}^{2}\right)$.

$c_{\perp}$ : Coeficiente de presión global para las fuerzas perpendiculares al andamio (de valor 0,9 ).

$A_{\mathrm{II}}$ : Proyección de la superficie total de todos los elementos redondos y planos, sobre un plano paralelo a la fachada (en $\left.\mathrm{m}^{2}\right)$. $A_{\text {IIs }}$ : Proyección de la superficie total de las secciones transversales particulares (las no incluidas en $A_{\mathrm{II}}$ ) sobre un plano paralelo a la fachada $\left(\mathrm{en}^{2}\right)$.

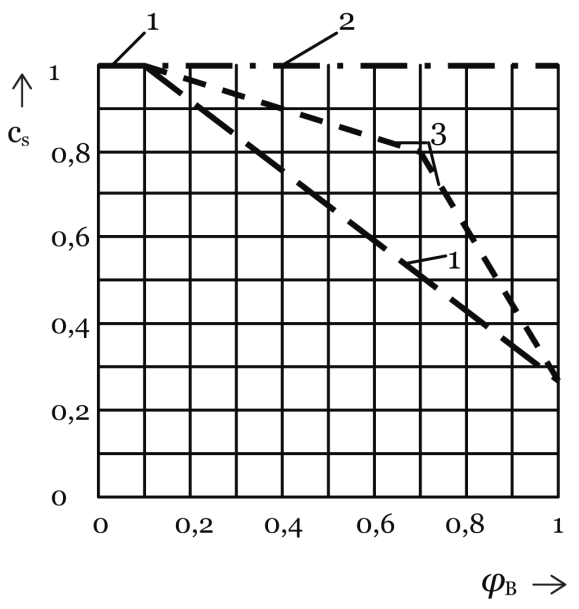

\section{LEYENDA:}

1 redes en ambas direcciones, paralela y normal; 2 lonas en ambas direcciones, paralela y normal; 3 lonas, pero sólo para el cálculo de las fuerzas de anclaje normales a la fachada; $c_{\mathrm{S}}$ coeficiente del sitio; $\varphi_{\mathrm{B}}$ relación solidez 
$c_{f}$ : Coeficiente de presión para las secciones transversales.

Como coeficientes parciales de seguridad para solicitaciones, $\gamma_{f}$, para cargas de viento, se establecen los valores de 1,50 en estados límites últimos y 1,00 en estados límites de servicio.

La norma UNE-EN 12810-1 indica que para configuraciones del sistema sin recubrimiento debe existir una zona libre de anclajes a fachada de 3,8 $\mathrm{m}$ de altura, por encima y por debajo del nivel anclado. Este requisito no es aplicable donde existan componentes auxiliares, como vigas puente.

En la Figura 8 (de la norma UNE-EN 12810-1) se dan ejemplos para modelos típicos de anclaje. La norma indica que es preferible que la zona libre de anclajes esté al menos a dos veces de la distancia entre los niveles de trabajo. El objeto de esta prescripción es asegurar que el andamio tiene suficiente resistencia total en el diseño.

Los amarres del andamio a la fachada deben realizarse cuando la estructura alcance el nivel de amarre previsto en el proyecto. Los amarres trasladan a la fachada del edificio todas las fuerzas horizontales que actúan sobre el andamio. En ningún caso los amarres deberían soportar acciones verticales.

Los dos tipos de amarres más comúnmente empleados son:

- Amarres mediante tacos (anclajes). Existe una tendencia generalizada por parte de los montadores a utilizar tacos de expansión, por su mayor facilidad de colocación. Se debe estudiar previamente el material base que constituye la fachada. Cuando el anclaje se realiza sobre obras de fábrica, lo más adecuado, a pesar de ser una instalación más lenta y compleja, es utilizar tacos químicos con vaina de malla metálica e inyección de resina, lo que proporciona una mayor superficie de reparto, disminuyendo la fuerza individual que solicita a cada anclaje.

- Amarres a huecos mediante husillos, puntales o elementos similares. El cálculo de la carga admisible de este tipo de amarre es más complejo que el anterior. Además en este caso es necesario el reapriete sistemático y continuado. También es necesario colocar piezas de contrachapado entre husillos y mochetas para absorber las dilataciones.

\section{EJEMPLO DE CÁLCULO DE LOS ANCLAJES DE UN ANDAMIO A FACHADA}

A modo de ejemplo se va a calcular un andamio de fachada para rehabilitar edificios. Las dimensiones del andamio son $24 \mathrm{~m}$ de longitud y $16 \mathrm{~m}$ de altura. El andamio está formado por elementos tipo marco de $70 \mathrm{~cm}$ de ancho y, cuando está colocado, se separa de la fachada $30 \mathrm{~cm}$. El andamio está revestido por lona. En la Figura 9 se muestra el esquema general del andamio.
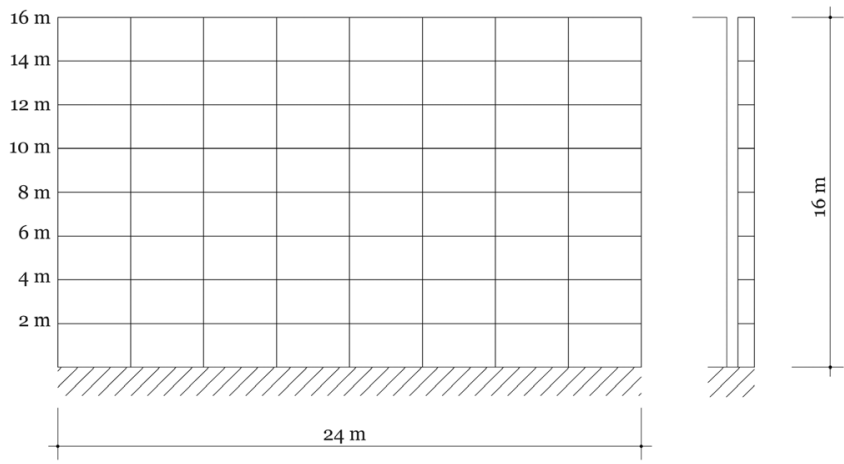

Figura 9. Esquema general del andamio.

Las cargas horizontales perpendiculares al plano del andamio producen un efecto de tracción en los amarres del andamio a fachada. La zona más desfavorable es la superior, donde la carga producida por el viento es mayor. La combinación de cargas más desfavorable es la hipótesis II, donde la acción del viento es máxima.

Las cargas horizontales paralelas al plano del andamio se resisten con los arriostramientos, el elemento más desfavorable es el situado en el nivel inferior, donde se suman las acciones horizontales de los niveles superiores. La combinación de cargas más desfavorable es la hipótesis II, donde la acción del viento es máxima.

\subsection{Acciones horizontales}

Se calcularan las acciones horizontales producidas por el viento que actúan sobre toda la estructura del andamio.

Acción del viento.

La fuerza resultante del viento se obtiene a partir de la ecuación [8]:

$$
F=c_{s} \sum_{i}\left(c_{f, i} \cdot A_{i} \cdot q_{i}\right)
$$

Presión del viento

La hipótesis II es la que conduce a la máxima fuerza de viento.

Hipótesis II. La condición de fuera de servicio.

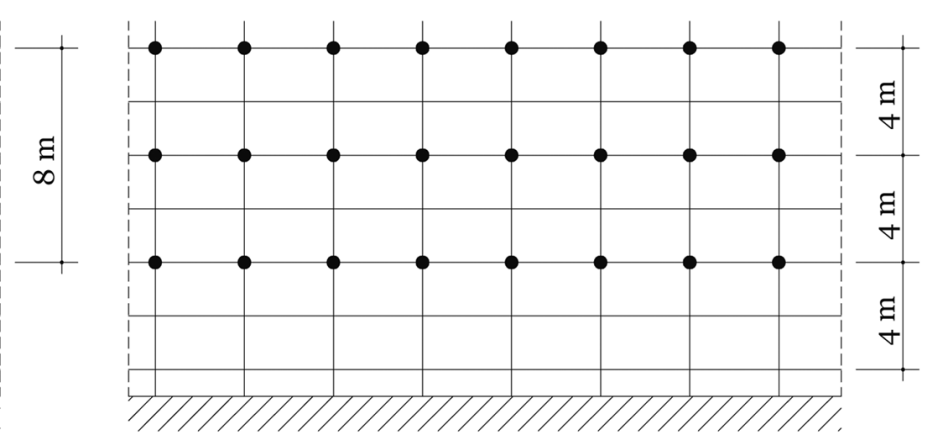

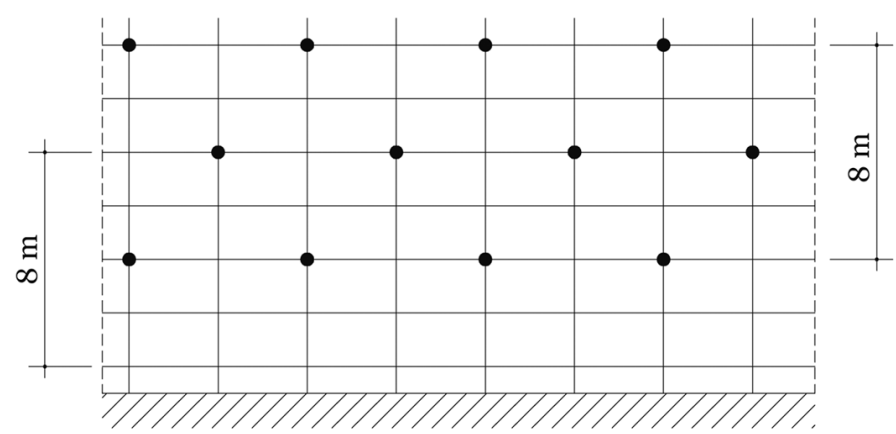

Figura 8. Ejemplos de modelos típicos de anclaje. 
La presión es variable, desde $800 \mathrm{~N} / \mathrm{mm}^{2}$ (altura $0,0 \mathrm{~m}$ ) hasta $1100 \mathrm{~N} / \mathrm{mm}^{2}$ (altura 24,0 m). Para una altura dada y, la presión $q(y)$ se obtiene de la siguiente ecuación [9]:

$$
q(y)=800+\frac{1100-800}{24} y=800+12,5 y
$$

Con $y$ en $m$ y $q(y)$ en $\mathrm{N} / \mathrm{mm}^{2}$.

La presión del viento desde el primer nivel (2,0 m) hasta la altura máxima del andamio $(16,0 \mathrm{~m})$ y a cada $2,0 \mathrm{~m}$ se indica en la Tabla 2.

Tabla 2

Presión debida al viento.

\begin{tabular}{|l|c|c|c|c|c|c|c|c|}
\hline $\mathrm{y}(\mathrm{m})$ & 2,0 & 4,0 & 6,0 & 8,0 & 10,0 & 12,0 & 14,0 & 16,0 \\
\hline $\mathrm{q}\left(\mathrm{N} / \mathrm{mm}^{2}\right)$ & 825 & 850 & 875 & 900 & 925 & 950 & 975 & 1000 \\
\hline
\end{tabular}

5.1.1. Viento paralelo a la fachada

Superficie expuesta al viento.

Coincide con la superficie del recubrimiento.

Superficie expuesta al viento por nivel [10]:

$$
A=0,70 \cdot 2,00=1,40 \mathrm{~m}^{2}
$$

Coeficiente del sitio.

Su valor es $c_{s}=1,0$ (gráfico de la Figura 5).

Coeficiente de fuerza aerodinámica.

Su valor es $c_{f}=0,1$ (Tabla 1$)$.

El valor de la fuerza total debida al viento paralelo a la fachada y por cada nivel se obtiene como el producto de los coeficientes de sitio y de fuerza aerodinámica por el área y por la presión. En la Tabla 3 se indican los valores sin mayorar correspondientes a la fuerza de viento paralelo a fachada y por cada 2,00 $\mathrm{m}$ de altura de andamio.
Tabla 3.

\begin{tabular}{|c|c|c|c|c|c|c|c|c|}
\hline (m & 2,0 & 4,0 & 6,0 & 8,0 & 10,0 & 12,0 & 14,0 & 16,0 \\
\hline$F(\mathrm{~N})$ & 116 & 119 & 123 & 126 & 130 & 133 & 137 & 14 \\
\hline
\end{tabular}

Fuerza sin mayorar debida al viento paralelo a fachada.

5.1.2. Viento perpendicular a fachada

Superficie expuesta al viento.

Coincide con la superficie del recubrimiento.

Superficie expuesta al viento por nivel [11]:

$$
A=24, \mathrm{o} \cdot 2,00=48,0 \mathrm{~m}^{2}
$$

Coeficiente del sitio.

Su valor es $c_{s}=1,0$ (gráfico de la Figura 5).

Coeficiente de fuerza aerodinámica.

Su valor es $c_{\mathrm{f}}=1,3$ (Tabla 1 ).

El valor de la fuerza total debida al viento perpendicular a la fachada y por cada nivel se obtiene como el producto de los coeficientes de sitio y de fuerza aerodinámica por el área y por la presión. En la Tabla 4 se indican los valores sin mayorar correspondientes a la fuerza de viento perpendicular a fachada y por cada 2,00 $\mathrm{m}$ de altura de andamio.

Tabla 4.

Fuerza sin mayorar debida al viento perpendicular a fachada.

\begin{tabular}{|c|c|c|c|c|c|c|c|c|}
\hline$y(\mathrm{~m})$ & 2,0 & 4,0 & 6,0 & 8,0 & 10,0 & 12,0 & 14,0 & 16,0 \\
\hline$F(\mathrm{~N})$ & 51480 & 53040 & 54600 & 56160 & 57720 & 59280 & 60840 & 62400 \\
\hline
\end{tabular}

El viento perpendicular a fachada provoca una fuerza horizontal que deben resistir los amarres del andamio a la fachada. Si se disponen los amarres indicados en la Figura 10, los puntos más desfavorables son los situados a más altura, donde la fuerza provocada por el viento es mayor.

Se van a comprobar dos situaciones:
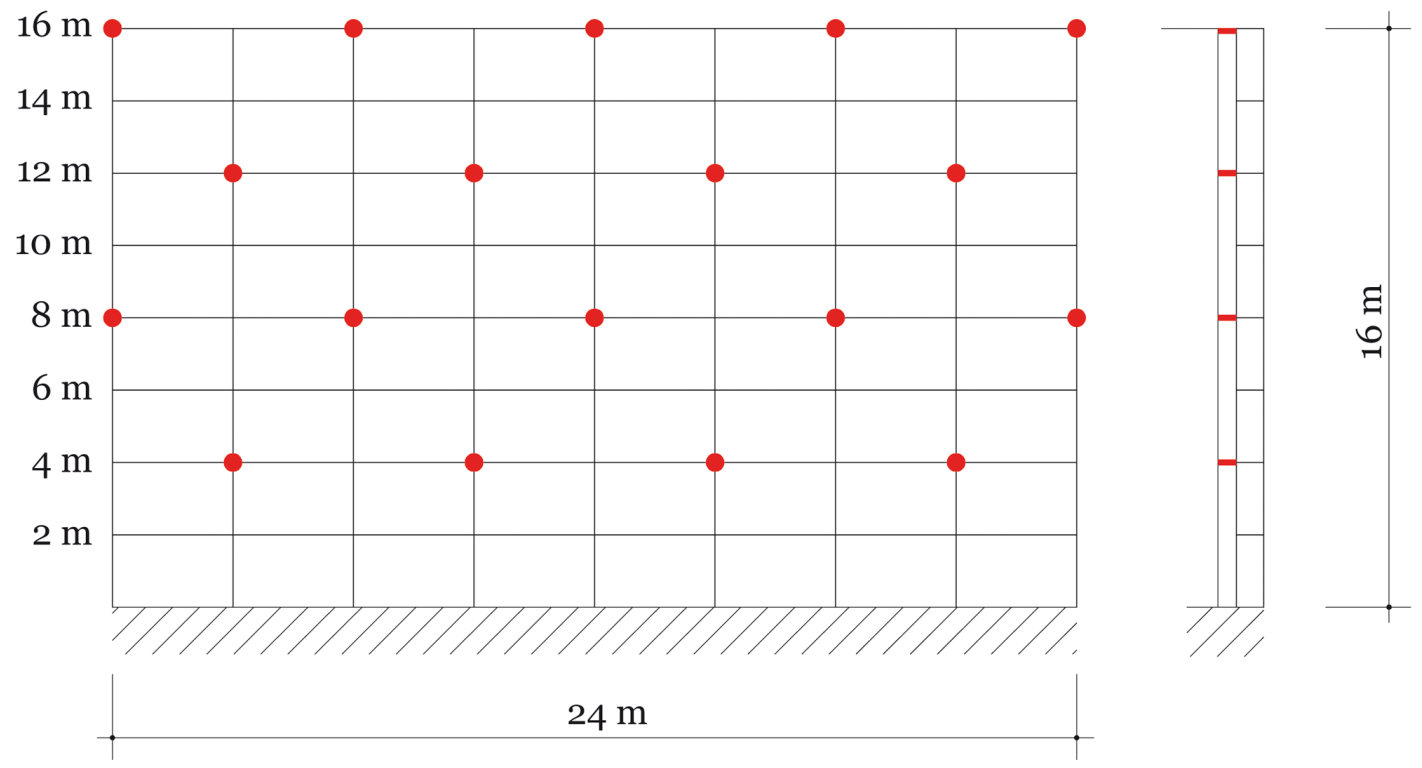

Figura 10. Disposición de los amarres. 
- La fila de amarres situada al nivel 16,0 m, correspondiente a la máxima fuerza horizontal.

- La fila de amarres situada al nivel 12,o m, donde la fuerza es menor pero el área de influencia es mayor.

Nivel 16,o m (5 amarres)

Carga total:

$$
Q_{t}=1,5\left(62400+\frac{60840}{2}\right)=139230 \mathrm{~N}
$$

Carga por amarre:

$$
Q=\frac{139230}{5}=27846 \mathrm{~N}
$$

Nivel 12,o m (4 amarres)

Carga total:

$$
Q_{t}=1,5\left(\frac{60840}{2}+59280+\frac{57720}{2}\right)=177840 \mathrm{~N}
$$

Carga por amarre:

$$
Q=\frac{177840}{4}=4446 \mathrm{oN}
$$

Con la disposición de amarres de la Figura 10, cada amarre a fachada debe ser capaz de resistir una fuerza a tracción de $44460 \mathrm{~N}$.

Este valor está muy por encima de los valores de resistencia a arrancamiento obtenidos experimentalmente sobre fábricas de albañilería por lo que habría que utilizar un mayor número de anclajes o usar anclajes de otro tipo.

La metodología del análisis empleada en este trabajo puede extrapolarse a la evaluación de anclajes de otros elementos empleados como PC o MAE. Así por ejemplo, en el caso de barandillas provisionales de seguridad empleadas en obras de restauración de fábricas de albañilería, cuando los postes van soldados a placas metálicas recibidas con anclajes a las fábricas, la solicitación sobre la placa cuando el poste es de un metro de altura sería de $0,72 \mathrm{kN} \cdot \mathrm{m}$ (corresponde al momento producido por una carga horizontal de $0,72 \mathrm{kN} \cdot \mathrm{m}$ y un brazo de palanca de $1 \mathrm{~m}$ ). Si la placa se recibe mediante 4 anclajes simétricos separados verticalmente $10 \mathrm{~cm}$, la fuerza de arrancamiento sería de $7,2 \mathrm{kN}$, obtenida como el cociente entre el momento solicitación y la distancia vertical entre anclajes. Esto supone una fuerza de arrancamiento por anclaje de 3,6 kN. Este valor no posee ningún coeficiente de seguridad. Con un coeficiente de seguridad de 2 , se obtiene una fuerza de arrancamiento superior a la obtenida experimentalmente. Una solución posible podría ser aumentar la distancia vertical entre los anclajes.

\section{CONCLUSIONES}

La fuerza de arrancamiento de anclajes de plástico a obras de albañilería no se conoce con la precisión necesaria para abordar el dimensionamiento de andamios.

En fábricas de albañilería de ladrillo macizo construidas en Madrid en la década de 1930 se han obtenido valores experimentales de arrancamiento de cáncamos roscados a tacos de plástico de 6,2 kN. Estos valores concuerdan con los obtenidos por otros autores $(4, \mathrm{o} \mathrm{kN}-5,7 \mathrm{kN})$.

La disposición de anclajes tipo que ofrecen las normas UNEEN queda totalmente del lado de la inseguridad para andamios de ocho alturas revestidos con lona cuando se usan tacos de plástico. En el ejemplo desarrollado en este trabajo, que representa un caso típico, la carga de cálculo de cada amarre alcanza $44,5 \mathrm{kN}$, muy por encima del valor de $6,2 \mathrm{kN}$, obtenido experimentalmente. En estos casos es necesario aumentar el número de anclajes o cambiar la tipología de anclaje.

\section{AGRADECIMIENTOS}

Los autores agradecen el apoyo de la empresa PROIESCON, necesario para realizar los ensayos de arrancamiento.

\section{REFERENCIAS}

(1) FIB. (2007). Manuel del' échafaudage. Paris: Syndicat Français del l'echafaudage du coffrage et de l' etaiement.

(2) Tamborero, J.M. (2004). Montaje y utilización de los andamios perimetrales fijos. Revista del INSHT, (32): 5-18.

(3) OSALAN. (2003). Guía práctica de andamios tubulares apoyados. Gobierno Vasco.

(4) García, J., Irles, R., Pomares, J.C. (2010, 26-29 de octubre). Mechanical behaviour of metal ties for security nets and IPE's. En XXXVII IAHS World Congress on Housing. Santander, España.

(5) García, J., Irles, R., Segovia, E., Pomares, J.C. (2008, 24-27 de noviembre). Definición de ensayos a realizar sobre anclajes de seguridad en redes y EPI's. En IV Congreso de la Asociación Científico-Técnica del Hormigón Estructural. Valencia, España.

(6) Cobo, A., González-Yunta, F., González-Rodrigo, S., González García, M.N. (2011, 16-18 de noviembre). Estudio de anclajes de andamios a fábricas de albañilería. En Workshop Investigación e Innovación en protecciones colectivas y medios auxiliares de edificación. Madrid, España.

(7) CEB. (1994). Fastenings to concrete and masonry structures. State of the art report. London: Thomas Telford Services Ltd.

(8) Fischerwerke. (1988). Untersuchungen an Rahmendübel $S$-RS in verschiedenen Mauerwerksarten (Auszug aus dem Prüfbericht).

(9) FMPA. (1977). Zulassungsversuche an Fischer S 10 H-R Dübeln in Mauerwerk aus Kreuzlochziegeln. Report S 13 oo8-1.

(10) FMPA. (1977). Untersuchungen des Tragverhaltens von Fischer-Gasbetondübeln GB 1O, GB 14. Report S12 976.

(11) Planck, A. (1977). Bautechnische Einflüsse auf auf die Tragfähigkeit von Kunststoffdübeln in Mauerwerk. BaumaschineBautechnik, 6: 406-416.

(12) Ministerio de Empleo y Seguridad Social. (2012, 15 de marzo). Resolución de 28 de febrero de 2012, de la Dirección General de Empleo, por la que se registra y publica el V Convenio colectivo del sector de la construcción. Boletín Oficial del Estado, (64): 23837-23967. 
(13) AENOR. (2003). UNE-EN 12810-1: 2003. Andamios de fachada de componentes prefabricados. Parte 1: Especificaciones de los productos. Asociación Española de Normalización (AENOR).

(14) AENOR. (2003). UNE-EN 12810-2: 2003. Andamios de fachada de componentes prefabricados. Parte 2: Métodos particulares de diseño estructural. Asociación Española de Normalización (AENOR).

(15) AENOR. (2003). UNE-EN 12811-1: 2003. Equipamiento para trabajos temporales de obra. Parte 1: Andamios. Requisitos de comportamiento y de diseño general. Asociación Española de Normalización (AENOR).

(16) AENOR. (2004). UNE-EN 12811-2: 2004. Equipamiento para trabajos temporales de obra. Parte 2: Información sobre los materiales. Asociación Española de Normalización (AENOR).

(17) AENOR. (2002). UNE-EN 12811-3: 2002. Equipamiento para trabajos temporales de obra. Parte 3: Ensayos de carga. Asociación Española de Normalización (AENOR).

(18) AENOR. (1998). Andamios de servicios y de trabajo, con elementos prefabricados. Materiales, medidas, cargas de proyecto y requisitos de seguridad. Asociación Española de Normalización (AENOR). 